\title{
Low-energy $\boldsymbol{\eta}$-nuclear scattering parametres vs. binding properties in light nuclei *
}

\author{
J. A. Niskanen \\ Helsinki Institute of Physics, PO Box 64, FIN-00014 University of Helsinki, \\ Finland
}

The connection of the binding energy and width of possible bound $\eta$ mesic states is given to the complex scattering length for $s$-states in the hope that, with knowledge of the final state interaction this could be useful in searches of these states. In spite of the strong direct potential dependence of both observables this connection is seen to be very model independent even for various nuclei once the influence of also the effective range is considered. The importance of this term is pointed also for data analyses. Although the nucleus considered here is ${ }^{12} \mathrm{C}$, extension to other nuclei is implied in the background work.

PACS numbers: 21.85.+d; 24.10.Ht; 13.75.-n

\section{Introduction}

As discussed in some other talks in this symposium, low-energy final state scattering parametres have been the target of intense analyses of $\eta$ meson production in recent years. Mostly the investigations aim at the extraction of the complex scattering length $a$, where the real part is connected to the existence or nonexistence of a bound state. The definition common in meson physics

$$
q \cot \delta=\frac{1}{a}+\frac{1}{2} r_{0} q^{2}
$$

requires a negative real part and $\left|a_{\mathrm{R}}\right|>a_{\mathrm{I}}$ (more precisely including also the effective range $\mathcal{R}\left[a^{3}\left(a^{*}-r_{0}^{*}\right)\right]>0$ [1, 2] $)$. However, unfortunately the scattering cross section data alone cannot give just the sign of this quantity.

\footnotetext{
* Presented at II International Symposium on Mesic Nuclei, Kraków, Poland, Septem-
} ber 23,2013 
Nevertheless, the magnitude of the energy of a bound state (or a virtual state) can be estimated from final state interaction (FSI) effects, though the existence cannot. This was the original idea of Ref. [1] studying the relation in ${ }^{3} \mathrm{He}$ provided a bound state would exist. This work was recently extended to heavier nuclei [2], where the existence of bound states is less controversial and experiments are being performed (see e.g. talks by Gal, Machner and Wilkin in these proceedings).

Particularly suggestive is the possibly rapid change of the scattering lengths in ${ }^{3} \mathrm{He}$ and ${ }^{4} \mathrm{He}$ discussed by Machner at the first symposium of this series [3]. There the latter scattering length appears significantly smaller than the large $a_{3} \mathrm{He}$, which would hint to a change of the sign (of the real part), since one would expect more attraction in the heavier nucleus and thus either even larger scattering length (for the nonbinding situation) or a binding strength with undetermined size of the cross section and scattering length. However, the results seem to depend strongly on whether they are obtained with or without the effective range term of the low-energy expansion. This is discussed in subsec. 3.2

The aim of this talk is to review and summarize the numerical relation of the complex binding energy to the complex scattering length and effective range. At the end some speculative extrapolation possibility is shortly played with.

\section{Formalism}

The basic idea is to employ various nuclear charge distributions $\rho(r)$ in simple optical potentials

$$
V_{\mathrm{opt}}=-4 \pi\left(V_{\mathrm{R}}+i V_{\mathrm{I}}\right) \rho(r) /\left(2 \mu_{\eta N}\right)
$$

where for each nucleus the strength parameters $\left(V_{\mathrm{R}}, V_{\mathrm{I}}\right)$ are freely varied to get a sufficient coverage to present the binding energy and width as contours in the $\left(a_{\mathrm{R}}, a_{\mathrm{I}}\right)$ plane. The normalization of the profiles shown in Fig. 11 is chosen to the mass number as $4 \pi \int_{0}^{\infty} \rho(r) r^{2} d r=A$, so that in the simple impulse approximation optical model the strength $\left(V_{\mathrm{R}}, V_{\mathrm{I}}\right)$ would correspond to the elementary scattering length $a_{\eta N}$ ( $r$ given in fm). However, it should be stressed that no claim is made about any absolute strength of the potential as in microscopic model works. The main purpose is to get the numerical connection of binding energies and widths to the scattering parameters, so that if the latter can be extracted from data, then a preliminary estimate could be obtained for the former. Possibly this would be useful for planning experiments. In the spirit of the shape independence of $N N$ forces, one might expect a more density profile independent connection 


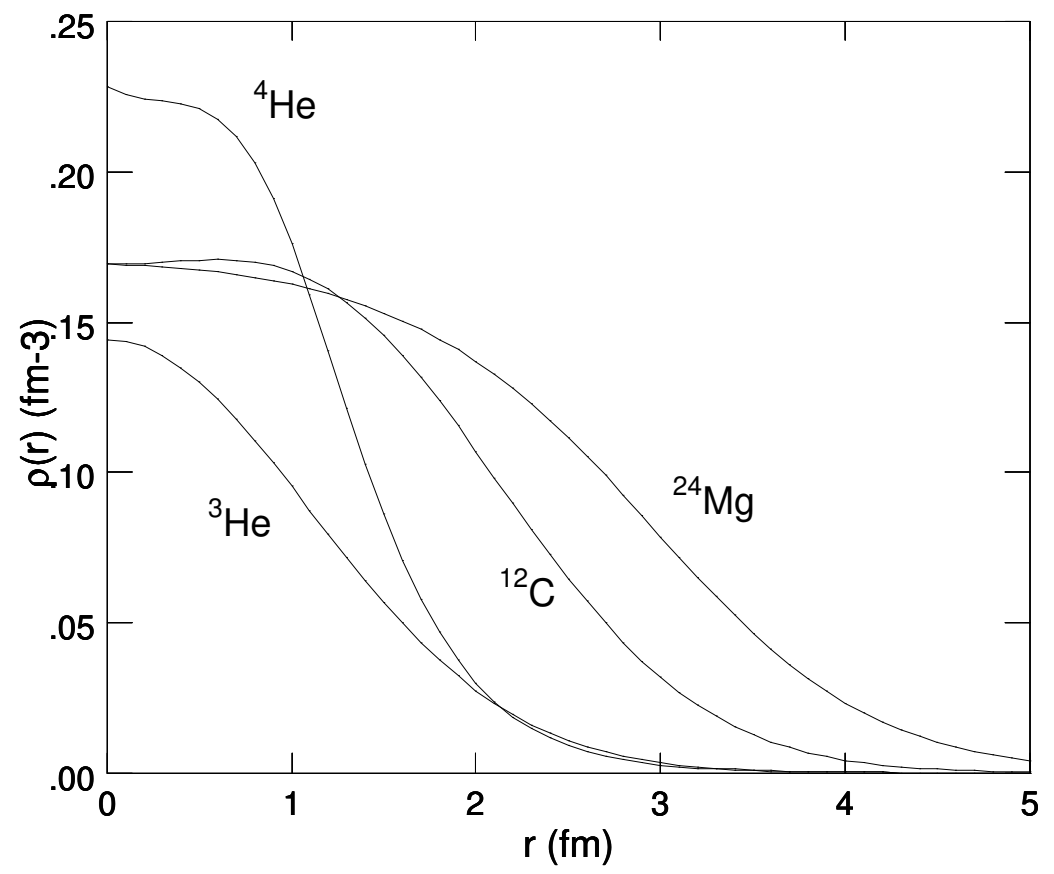

Fig. 1. The density profiles of the nuclei used in Refs. [1] and [2].

between these quantities than in the direct relation to the potential of either of them.

If the effective range term is included, then also the energy relation should include this and the low (complex) energy relation would be

$$
1 / a=-\sqrt{-2 \mu_{\eta A} E / \hbar^{2}}-r_{0} \mu_{\eta A} E / \hbar^{2}
$$

with $\mu_{\eta A}$ the reduced mass of the system. In comparison with the actual exact results from solving equations of motion this relation was found to be much more accurate than keeping the first term alone. For binding energies up to $|E| \approx 10 \mathrm{MeV}$ and even beyond the accuracy of eq. 3 was a few percent for the real part and about ten percent for the imaginary $\left(E\right.$ and $r_{0}$ taken from the calculation). This success can be attributed to the inclusion of a non-zero effective range $r_{0}$ and can be considered as an indication of the necessity of its inclusion also in data analyses. 


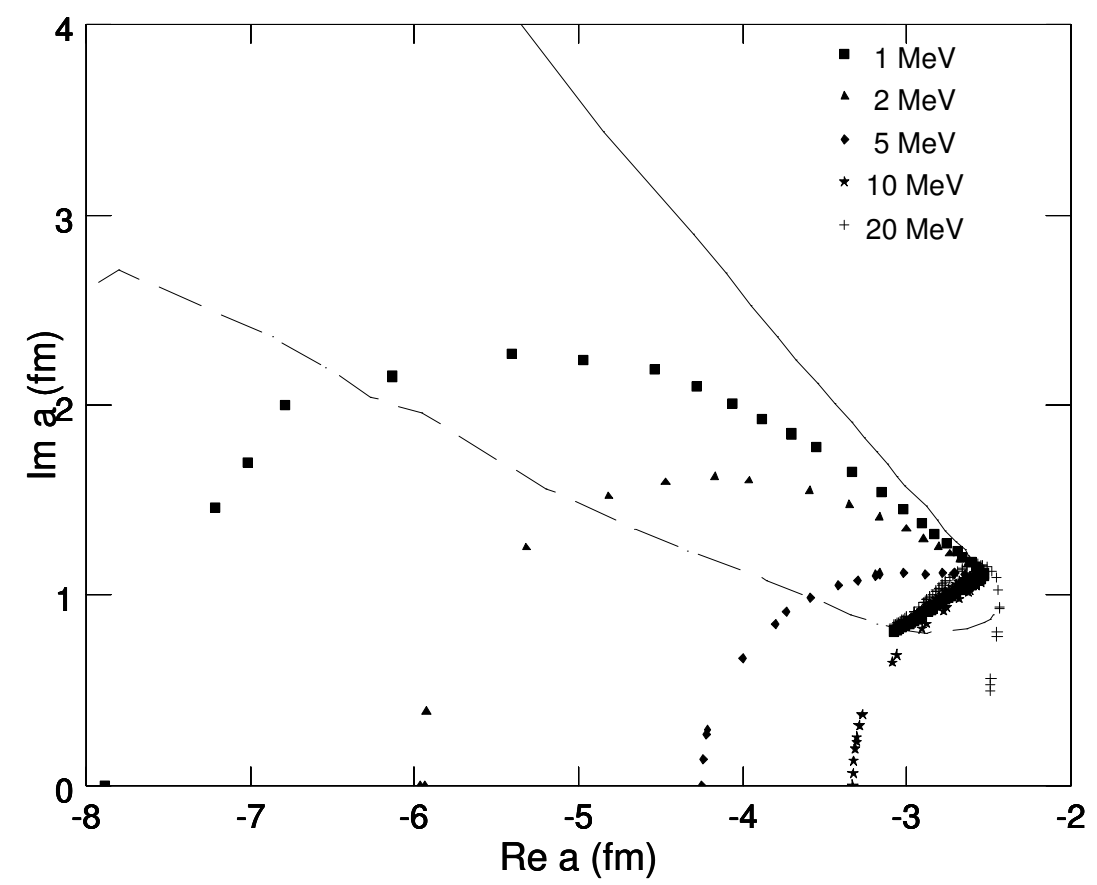

Fig. 2. The $s$-wave binding energy $E_{\mathrm{B}}=-E_{\mathrm{R}}$ contours for $1,2,5,10$ and $20 \mathrm{MeV}$ in the complex $\left(a_{\mathrm{R}}, a_{\mathrm{I}}\right)$ plane. The solid line shows the zero energy, i.e. above it there is no binding. Below the dashed line $\left|E_{\mathrm{R}}\right|>\left|E_{\mathrm{I}}\right|$.

\section{Numerical results}

\subsection{Case of ${ }^{12} \mathrm{C}$}

Going to heavier nuclei from ${ }^{3} \mathrm{He}$ carbon may be a representative compromise between the experimentally studied helium and magnesium isotopes. Fig. 2 shows the binding energy contours for five choices up to $20 \mathrm{MeV}$. Also shown are the zero energy limit and the borderline where $\left|E_{\mathrm{R}}\right|>\left|E_{\mathrm{I}}\right|$. The latter is of interest as a measure of actually distinguishing any peak. To facilitate the complete connection Fig. 3 shows also the contours of constant half-widths $\left(-E_{\mathrm{I}}\right)$.

The behaviour for a real potential is quite clear and understandable, but some peculiar features appear for strongly absorptive interactions. Absorption, described by the imaginary part, acts in the bound system like a repulsion eating away the wave function in the attractive region: less wave function $\longrightarrow$ less attraction. So for increasing absorption the strength of also the attractive real part must be increased to stay on the equal-energy con- 


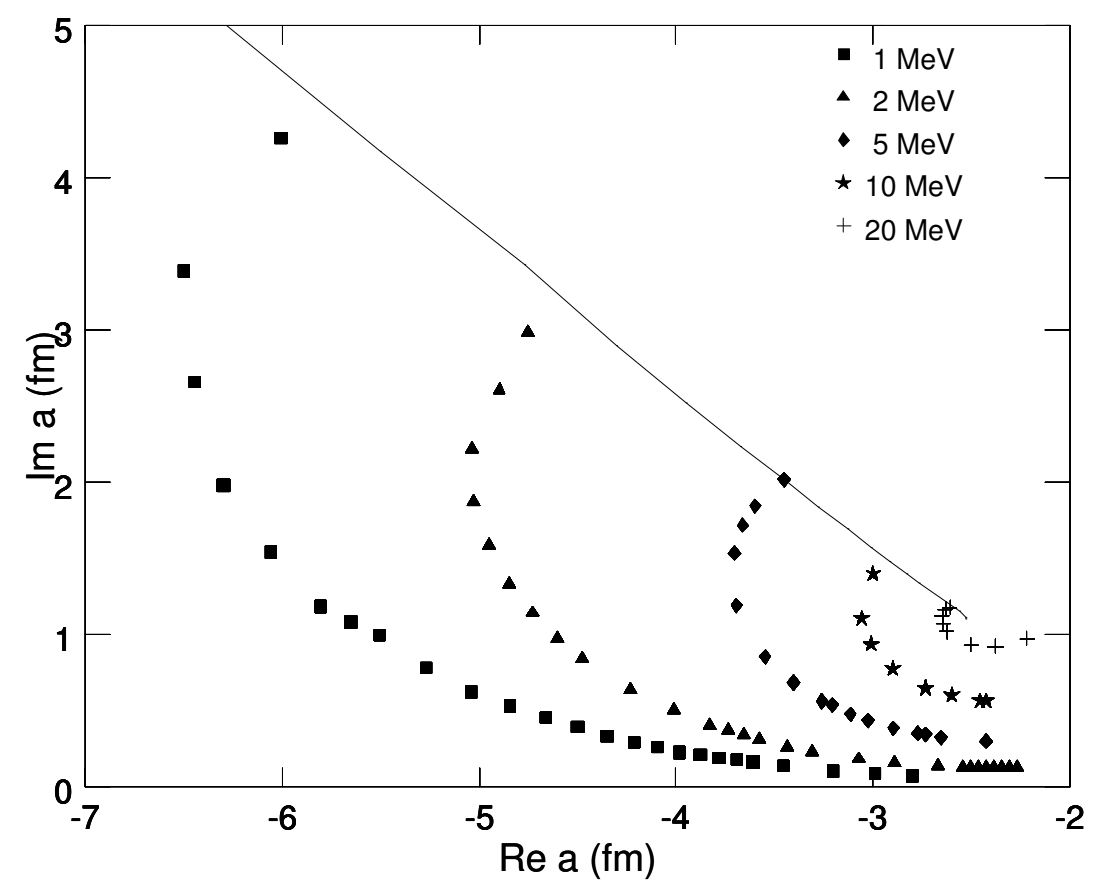

Fig. 3. The same as Fig. 2 but for the imaginary part of the bound state energies $-E_{\mathrm{I}}$, i.e. half widths.

tours. However, as shown in Ref. 2] the effect of the imaginary part of the potential saturates in the scattering length and $a_{\mathrm{I}}$ is not even monotonously increasing. Therefore, the calculated points for the equidistant mesh of the potential strength become denser and denser with increasing absorption (and stronger real part to remain at the same binding energy) and eventually the contours turn back to left. However, in this region the states are so wide, anyway, that they may not be of experimental interest. Furthermore, it may be worth noting that in the proximity of zero binding the widths tend to be large, significantly larger than the binding energy for the same $V_{\mathrm{R}}$ without imaginary part at all. Therefore, near threshold bound states may be hard to distinguish.

To get a more detailed picture of that behaviour and the general trend of increasing binding energies Fig. 4 shows a magnified detail of Fig. 2. It can be seen that the "weak potential branch" comes lower and lower in the $a_{\mathrm{I}}$ direction and the opening angle between the "branches" decreases until they cross over at about $9 \mathrm{MeV}$ binding. So the $10 \mathrm{MeV}$ contour below the 


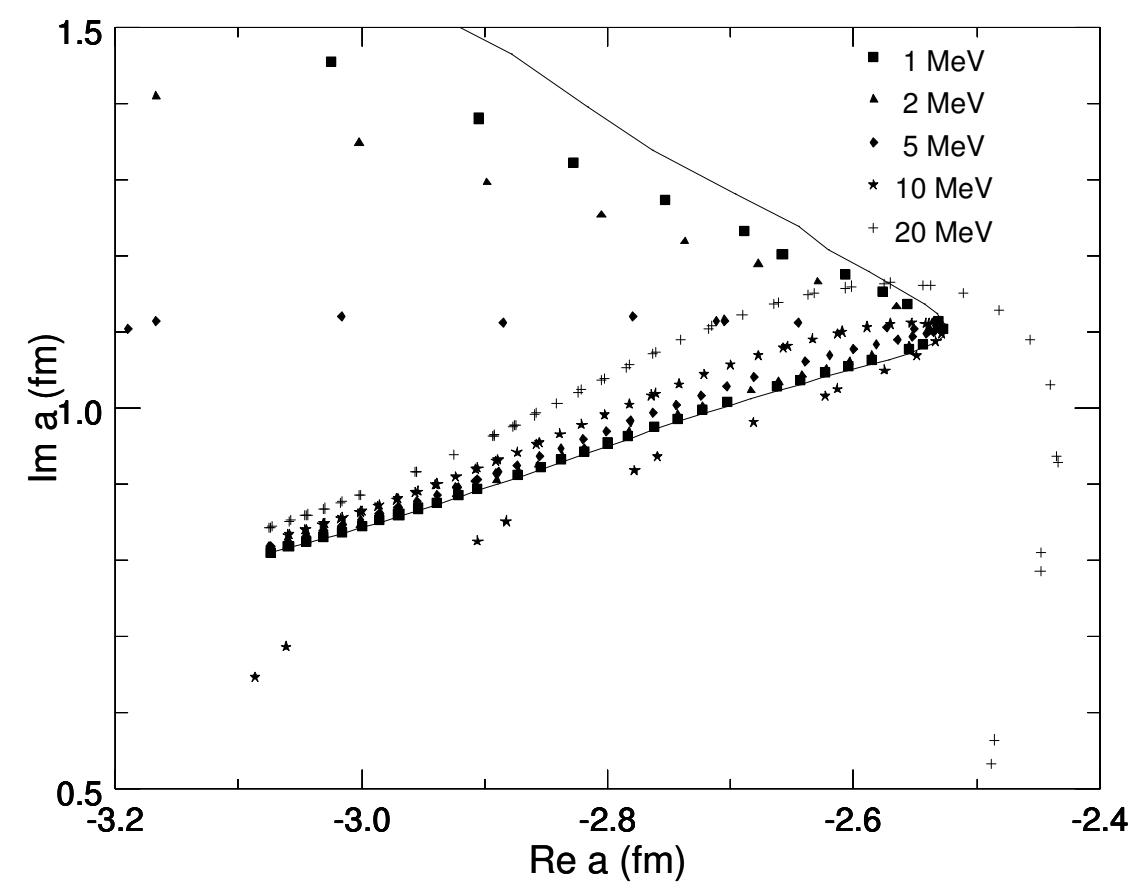

Fig. 4. Magnified detail of Fig. 2

formal strong potential zero line belongs actually to the weaker couplings and $20 \mathrm{MeV}$ has gone even further.

The behaviour seen in Figs. 2 and 3 is very similar to that of ${ }^{3} \mathrm{He}[1$ and also ${ }^{4} \mathrm{He}$ and ${ }^{24} \mathrm{Mg}[2$ especially for large $|a|(>4)$ and "weak potential branch" dictated mainly by just the binding energy term in Eq. 3. The next section discusses the potential dependence through the effective range, which in turn depends naturally on the size of the nucleus.

\subsection{Significance of the effective range}

From relation 3 it is clear that at least with "realish" (dominantly real) quantities for a negative $E$ (consequently also negative $a$ ) and a positive effective range the two terms on the left hand side tend to cancel making $1 / a$ smaller and $a$ bigger. Due to this cancellation $a$ is quite sensitive on the inclusion of the second term. This expectation is actually seen in drastic changes of the data analyses from Refs. [4, 5, 6] giving the scattering lengths shown in Table1. Clearly leaving the effective range out may give scattering lengths even less than one half of their true values and consequently grossly 
Table 1. Scattering lengths in fm from data with and without the effective range term.

\begin{tabular}{l|c|c|c} 
System & $a_{\mathrm{R}}$ & $a_{\text {I Ref. }}$ & \\
\hline$\eta\left({ }^{3} \mathrm{He}\right)$ & $\pm(2.9 \pm 2.7)$ & $3.2 \pm 1.8$ & {$[4]$} \\
$\eta\left({ }^{3} \mathrm{He}\right)$ & $\pm(10.7 \pm 0.9)$ & $1.5 \pm 2.6$ & {$[\underline{5}]$} \\
$\eta\left({ }^{4} \mathrm{He}\right)$ & $\pm(3.1 \pm 0.59$ & $0 \pm 0.5$ & {$[6]$} \\
$\eta\left({ }^{4} \mathrm{He}\right)$ & $\pm(6.2 \pm 1.9)$ & $0.01 \pm 6.5$ & {$[6]$} \\
\hline
\end{tabular}

overestimated binding energies. This is confirmed in the calculations and by Eq. 3 .

Consequently, it is clear that consistency in estimating the possible binding energy from the more advanced scattering analyses needs also the effective range term. If one takes for example the scattering lengths for $\eta^{4} \mathrm{He}$ above, leaving that away and making the standard estimate for possible binding energy, the first one (without $r_{0}$ ) would give about $4 \mathrm{MeV}$ and the second (with $\left.r_{0}\right) 1 \mathrm{MeV}$. However, from Fig. 2 the results for these two $a$ 's would be $10 \mathrm{MeV}$ and $2 \mathrm{MeV}$, correspondingly, the second being preferable. (The result for ${ }^{4} \mathrm{He}$, Fig. 8 of Ref. [2], is very nearly the same as ${ }^{12} \mathrm{C}$ for such weak binding due to shape independence. Practically only the region of the "turning point" tends to move left towards larger $\left|a_{\mathrm{R}}\right|$ with increasing $A$ and right for decreasing.)

Because of the apparent importance of the effective range Ref. [2] gives a parametrization of the complex $r_{0}$ in terms of the real and imaginary parts of the scattering length.

\subsection{Prospect of extrapolation}

Although the aim of this talk is not to promote a definite potential model, it may still be tempting to speculate by interpreting the strength parametres $V_{\mathrm{R}}$ and $V_{\mathrm{I}}$ in Eq. (2) to be the real and imaginary parts of the basic $\eta N$ scattering length $a_{\eta N}$ in a first order optical potential as done in e.g. Ref. [7. (Or they might represent some more general strengths perhaps extracted from microscopic nuclear models.) Then the strength $V_{\mathrm{R}}=0.33$ $\mathrm{fm}, V_{\mathrm{I}}=0 \mathrm{fm}$ would support a barely bound state in ${ }^{4} \mathrm{He}$, which would mean that all standard values of Re $a_{\eta N}$ ranging roughly between $0.4 \mathrm{fm}$ and $0.7 \mathrm{fm}$ could have the potential for binding in this nucleus. For this basic strength the binding energy in ${ }^{12} \mathrm{C}$ would be $8 \mathrm{MeV}$ and in ${ }^{24} \mathrm{Mg} 14$ $\mathrm{MeV}$. To produce a barely bound state in the three nuclei considered above the fitted strength $V_{\mathrm{R}}=0.86 \times A^{-0.7} \mathrm{fm}$ would be needed as shown by the squares and the dashed curve in Fig. 5 (also ${ }^{3} \mathrm{He}$ is shown). 


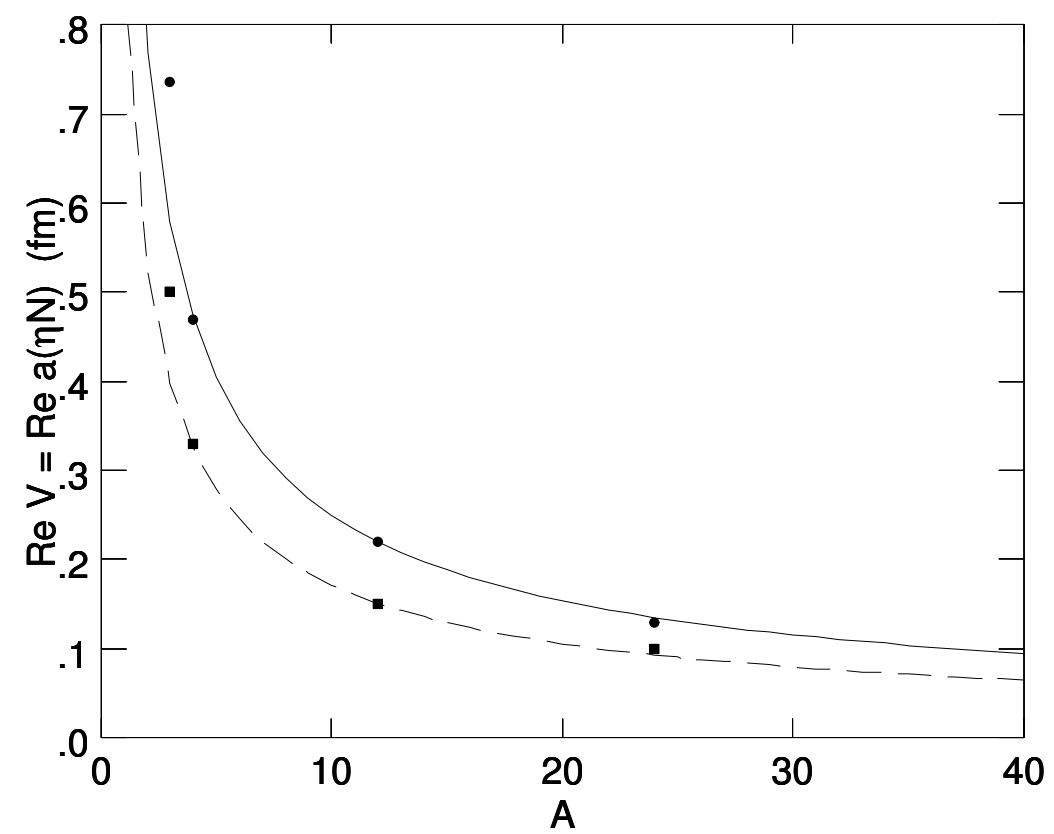

Fig. 5. Dependence of the binding limit on the mass number. Squares and dashed line for real potentials, dots and solid curve for $V_{\mathrm{I}}=V_{\mathrm{R}} / 2$.

However, $a_{\eta N}$ is complex and as another example, assuming more realistically $V_{\mathrm{I}}=0.5 V_{\mathrm{R}}$ (roughly true for most $a_{\eta N}$ in the literature), $V_{\mathrm{R}}=0.47$ fm would just bind the $\eta^{4} \mathrm{He}$ system and the corresponding binding energies would be $15 \mathrm{MeV}$ and $22 \mathrm{MeV}$ for ${ }^{12} \mathrm{C}$ and ${ }^{24} \mathrm{Mg}$, respectively. Their half-widths would be about the same as their binding energies; in the case of ${ }^{4} \mathrm{He}$ the half-width would be $12 \mathrm{MeV}$. In this case the barely binding strength could be parametrized as $V_{\mathrm{R}}=1.25 \times A^{-0.7} \mathrm{fm}$ (the circular dots and the solid curve). It may be of further interest to remind that the depth of a square well potential producing a zero binding energy should behave like $R^{-2} \propto A^{-2 / 3}$.

\section{Conclusion}

In this work a phenomenological connection between the low energy scattering length and the complex binding energy of possible eta-nuclear bound states has been studied in a simple but probably realistic model. The hope is that the results could be useful in searches of these bound states, 
if more easily accessible final state data are available to make predictions where to look for the states. The binding energies are explicitly presented as contours in the complex $a$ plane for the ${ }^{12} \mathrm{C}$ (also for ${ }^{4} \mathrm{He}$ and ${ }^{24} \mathrm{Mg}$ in Ref. [2]). It is seen that the connection is very closely the same and systematic for relatively different density profiles, from which it is easy to interpolate and even extrapolate to other nuclei.

The calculations suggest that for even relatively moderate values of the absorptive potential and of the imaginary parts of the scattering lengths, the states can be wide especially compared with the real depths of the states. In view of also many other theoretical results, starting from the elementary $\eta N$ scattering and predicting negative real parts for the scattering length but with rather large imaginary parts, the observation of such bound states might be difficult or even impossible. However, in the minireview [8] of the situation a reanalysis of the existing data on $\eta^{3} \mathrm{He}$ final states made very small values of the imaginary part appear plausible, so that also the possible bound states may not necessarily be as wide as most theoretical works would indicate.

In our work for $a_{\mathrm{I}}$ less than $2 \mathrm{fm}$ with $a_{\mathrm{R}}$ larger than, say, $5 \mathrm{fm} \mathrm{a}$ bound state should be recognizable. In the case of more likely smaller scattering lengths $a_{\mathrm{I}}<1 \mathrm{fm}$ would be necessary. For the assessment of possible distinguishable bound states Fig. 2 indicates the region where the half width is less than the binding energy. Experimentally in this respect the result $a_{\mathrm{R}}= \pm 6.2 \pm 1.9 \mathrm{fm}$ and $a_{\mathrm{I}}=0.001 \pm 6.5 \mathrm{fm}$ for ${ }^{4} \mathrm{He}$ of Ref. [6] is quite interesting and suggestive. If $\mid a_{\mathrm{R}}\left(\eta^{4} \mathrm{He} \mid\right.$ is really larger than for $\eta^{3} \mathrm{He}$ scattering, it is difficult to see how this could be true for a more attractive but still unbinding system.

The relation between $a$ and $E$ is very robust against potential differences even between different nuclides over a wide range once also the non-zero effective range is taken into account. Therefore, due to this shape independence one may trust the results to be valid by interpolation also for the $A=7$ nuclei of recent experimental interest [9].

I thank Pawel Moskal for the invitation and kind hospitality at the Symposium and HIP for a travel grant.

\section{REFERENCES}

[1] A. Sibirtsev, J. Haidenbauer, J. A. Niskanen, U. Meissner, Phys. Rev. C 70, 047001 (2004).

[2] J. A. Niskanen, H. Machner, Nucl. Phys. A 902, 40 (2013).

[3] H. Machner, Acta Phys. Polonica B 41, 2221 (2010). 
[4] J. Smyrski, et al., Phys. Lett. B 649 (2007) 258.

[5] T. Mersmann et al., Phys. Rev. Lett. 98 (2007) 242301.

[6] A. Budzanowski et al. (The GEM Collaboration) Nucl. Phys. A 821 (2009) 193.

[7] C. Wilkin, Phys. Rev. C 47 (1993) R938.

[8] A. Sibirtsev, J. Haidenbauer, C. Hanhart, and J. A. Niskanen, Eur. Phys. Journal A 22 (2004) 495.

[9] A. Budzanowski et al. (COSY-GEM collaboration), Phys. Rev. C 82 (2010) 041001R. 